\title{
Allosteric inhibition of hypoxia inducible factor-2 with small molecules
}

\author{
Thomas H Scheuermann ${ }^{1,2}$, Qiming Li', He-Wen Ma', Jason Key 1,2, Lei Zhang', Rui Chen ${ }^{3,4}$, \\ Joseph A Garcia ${ }^{3,4}$, Jacinth Naidoo', Jamie Longgood', Doug E Frantz', Uttam K Tambar', \\ Kevin H Gardner ${ }^{1,2 \star} \&$ Richard K Bruick ${ }^{1 \star}$
}

\begin{abstract}
Hypoxia inducible factors (HIFs) are heterodimeric transcription factors induced in many cancers where they frequently promote the expression of protumorigenic pathways. Though transcription factors are typically considered 'undruggable', the PAS-B domain of the HIF-2 $\alpha$ subunit contains a large cavity within its hydrophobic core that offers a unique foothold for smallmolecule regulation. Here we identify artificial ligands that bind within this pocket and characterize the resulting structural and functional changes caused by binding. Notably, these ligands antagonize HIF-2 heterodimerization and DNA-binding activity in vitro and in cultured cells, reducing HIF-2 target gene expression. Despite the high sequence identity between HIF-2 $\alpha$ and HIF-1 $\alpha$, these ligands are highly selective and do not affect HIF-1 function. These chemical tools establish the molecular basis for selective regulation of HIF-2, providing potential therapeutic opportunities to intervene in HIF-2-driven tumors, such as renal cell carcinomas.
\end{abstract}

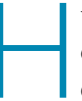
uman cells respond to hypoxia through the coordinated actions of the HIF family of transcription factors ${ }^{1}$. Assembled as heterodimers of an oxygen-sensitive subunit (HIF- $1 \alpha$, HIF- $2 \alpha$ or HIF- $3 \alpha$ ) and a dimerization partner (aryl hydrocarbon receptor nuclear translocator (ARNT) or HIF- $\beta$ ), these proteins control the expression of hundreds of genes that facilitate cellular adaptation and responses to low oxygen ${ }^{2,3}$. Although HIFs perform critical physiological functions ${ }^{1,4,5}$, increased amounts of these potent factors are highly correlated with the onset and progression of a variety of cancers $^{1}$. Indeed, several downstream targets of HIF are well-validated targets for anticancer therapies. However, there are potentially noteworthy advantages to directly antagonizing the HIF complexes themselves, and consequently their many downstream targets, as supported by experiments linking HIF ablation to impaired tumorigenesis ${ }^{6-8}$. As such, there is strong interest in the development of artificial compounds to regulate HIF function, to generate both basic research reagents and lead compounds for therapeutic development.

However, HIF presents a traditionally challenging target for pharmacological intervention: it is a large, intracellular protein complex without any active sites, which are typically used for small-molecule substrate binding. In addition, much of the transcription factor resides primarily in an extended conformation, further reducing the availability of potential ligand binding sites. However, both HIF subunits contain Per-ARNT-Sim (PAS) protein-protein interaction domains that contribute to the assembly of the HIF complex ${ }^{9,10}$ and the recruitment of coactivators ${ }^{11,12}$. These PAS domains are widely used as environmental sensors throughout biology, controlling activities of a diverse array of proteins ${ }^{13}$. Notably, such environmental sensing is often achieved by binding small-molecule cofactors within the core of a PAS domain, using ligand-induced allosteric changes to control the affinity for other protein elements bound to the outside surface ${ }^{14}$. Given the difficulties in directly and selectively antagonizing protein-protein interactions with small molecules ${ }^{15,16}$, exploiting such internal cavities offers potential advantages.
The PAS-B domain from HIF- $2 \alpha$ seems to be especially amenable to ligand-mediated allosteric regulation. This particular PAS domain contains a relatively large $\left(290 \AA^{3}\right)$ preformed cavity that can be occupied by either water or small molecules ${ }^{17,18}$. Using NMR-based screens of small-fragment libraries, we previously demonstrated that this site can be bound by small-molecule ligands with submicromolar affinities, inducing conformational changes that impair heterodimerization of isolated PAS-B domains in vitro ${ }^{18}$. Unfortunately, these molecules were not themselves suitable for further characterization in cultured cells, leading us to use a function-based high-throughput screen to survey a larger library of more complex compounds for inhibitors capable of disrupting an engineered HIF-2 PAS-B heterodimer.

Here we substantially advance these studies via the development of an improved small-molecule scaffold initially identified in this screen. Biophysical characterization of these compounds and a derivative optimized by medicinal chemistry approaches demonstrate specific, selective and efficacious binding within the internal cavity of HIF-2 $\alpha$ PAS-B. These compounds disrupt heterodimerization of the full-length HIF-2 transcription factor. Notably, these molecules function effectively as HIF-2 inhibitors in living cells, disrupting HIF-2 DNA binding and the transcription of its target genes. Moreover, these compounds are selective for the HIF-2 isoform and fail to antagonize HIF-1, whose highly related HIF- $1 \alpha$ subunit lacks a comparable ligand-binding site. These reagents provide an opportunity to delineate differences in HIF-1 and HIF-2 physiology and serve as an entry point for eventual selective therapeutic inactivation of HIF-2 in diseases ${ }^{19}$, including renal cell carcinomas ${ }^{20-22}$.

\section{RESULTS}

Identification of HIF-2 PAS-B dimerization antagonists

We previously identified ligands that bind the HIF- $2 \alpha$ PAS-B domain using solution NMR-based screening of a small library of almost 800 drug-like fragments ${ }^{17,18,23}$. Following medicinal chemistry optimization, some advanced lead compounds bound HIF- $2 \alpha$

\footnotetext{
'Department of Biochemistry, University of Texas Southwestern Medical Center, Dallas, Texas, USA. ${ }^{2}$ Department of Biophysics, University of Texas Southwestern Medical Center, Dallas, Texas, USA. ${ }^{3}$ Department of Medicine, Veterans Affairs North Texas Health Care System, Dallas, Texas, USA. 4Department of Internal Medicine, University of Texas Southwestern Medical Center, Dallas, Texas, USA. ^e-mail: Kevin.Gardner@utsouthwestern.edu orRichard.Bruick@utsouthwestern.edu
} 
with submicromolar dissociation constants. Despite their large size (molecular weight $\sim 300 \mathrm{Da}$ ), all of these ligands bound within an internal pocket $\left(290 \AA^{3}\right)$ completely buried within the HIF- $2 \alpha$ PAS-B domain ${ }^{17,18}$. Cavities of this size or larger are quite rare and are present in only $\sim 0.3 \%$ of over 32,000 high-resolution structures of comparably sized proteins or protein domains (Supplementary Results, Supplementary Fig. 1). Almost all of these cavities constitute ligand-binding sites within the apo forms of natural ligandbinding proteins. Consistent with a comparable functional role for the cavity in HIF-2 $\alpha$ PAS-B, our artificial ligands showed modest abilities to disrupt isolated PAS-PAS interactions in vitro ${ }^{18}$. However, their limited potency to do so limited further characterization in living cells.

To identify superior chemical scaffolds with the potential to antagonize HIF-2 activity in living cells, we developed an in vitro assay that assessed functional disruption of PAS-PAS interactions in a highthroughput screening (HTS) format. The isolated wild-type domains associate with $K_{\mathrm{D}} \sim 100 \mu \mathrm{M}$, precluding many protein-protein interaction assays. This interaction can be improved by more than 100fold by introducing mutations that enhance ionic interactions at the complex interface without altering other PAS features, including the HIF-2 $\alpha$ ligand-binding site ${ }^{18}$. These 'PAS- $\mathrm{B}^{\star}$ ' variants (HIF-2 $\alpha^{\mathrm{R} 247 \mathrm{E}}$ and ARNT ${ }^{\mathrm{E} 32 \mathrm{R}}$ ) were used in an amplified luminescent proximity homogeneous assay (AlphaScreen) to identify compounds capable of disrupting the stabilized heterodimer (Supplementary Fig. 2).

Using this HTS assay, over 200,000 compounds were individually interrogated for their ability to disrupt the HIF-2 $\alpha-$ ARNT PAS-B ${ }^{*}$ complex (Supplementary Table 1). The top 640 'hit' compounds, each of which decreased the luminescence proximity signal by over $3 \sigma$, were reassayed. Approximately $80 \%$ of these initial hits were validated, reflecting the high quality of this screen. However, a high number of these confirmed hits antagonized a key counterscreen designed to eliminate compounds that interfere with the AlphaScreen format itself. Once these nonspecific compounds were eliminated, fewer than 70 candidate disruptors of the HIF-2 $\alpha$-ARNT PAS- ${ }^{*}$ heterodimer remained. Subsequent titrations of a resupplied subset of these compounds revealed several compounds with standard dose-dependent behavior, with halfmaximum effective concentration $\left(\mathrm{IC}_{50}\right)$ values ranging between $0.3 \mu \mathrm{M}$ and $10 \mu \mathrm{M}$, as exemplified for compound $\mathbf{1}$ (Supplementary Fig. 3a,b). Notably, this class of compounds shared some structural features with amine-linked compounds identified from our NMRbased ligand binding screens $s^{17,18}$, leading us to further investigate their mode of action and potency.

In principle, the HIF- $2 \alpha$-ARNT PAS- ${ }^{*}$ heterodimer could be disrupted by small molecules that bind either of the two PAS-B subunits, either within their cores or at the $\beta$-sheet surfaces that mediate protein-protein interactions. On the basis of the similarity of 1 to compounds we previously observed in HIF-2 $\alpha$-ligand complexes $^{18,23}$, we anticipated that they might directly bind within the HIF- $2 \alpha$ PAS- $B^{*}$ domain. Using NMR spectroscopy to compare ${ }^{15} \mathrm{~N}-{ }^{-1} \mathrm{H}$ HSQC spectra of both PAS-B domains in the absence and presence of 1 , widespread ligand-induced spectral changes were observed for HIF-2 $\alpha$ PAS-B but not for the corresponding ARNT PAS-B subunit (Supplementary Fig. 3c). The slow exchange behavior for these chemical shift changes was consistent with low-micromolar or tighter dissociation constants. These binding affinities were quantified using isothermal titration calorimetry (ITC), reporting $K_{\mathrm{D}}=$ $1.1 \mu \mathrm{M}$ and a 1:1 stoichiometry (Supplementary Fig. 3d), though the high DMSO required to solubilize 1 most likely attenuated the true ligand affinity slightly ${ }^{17}$. In all, there was reasonable correlation between ligand affinity and the submicromolar $(\sim 0.4 \mu \mathrm{M}) \mathrm{IC}_{50}$ observed for heterodimer disruption (Supplementary Fig. 3b).

A medicinal chemistry effort was undertaken to understand the structure-activity relationships underlying the ability of 1 to disrupt HIF-2 $\alpha$-ARNT PAS-B ${ }^{\star}$ heterodimerization ${ }^{24}$. An improved a
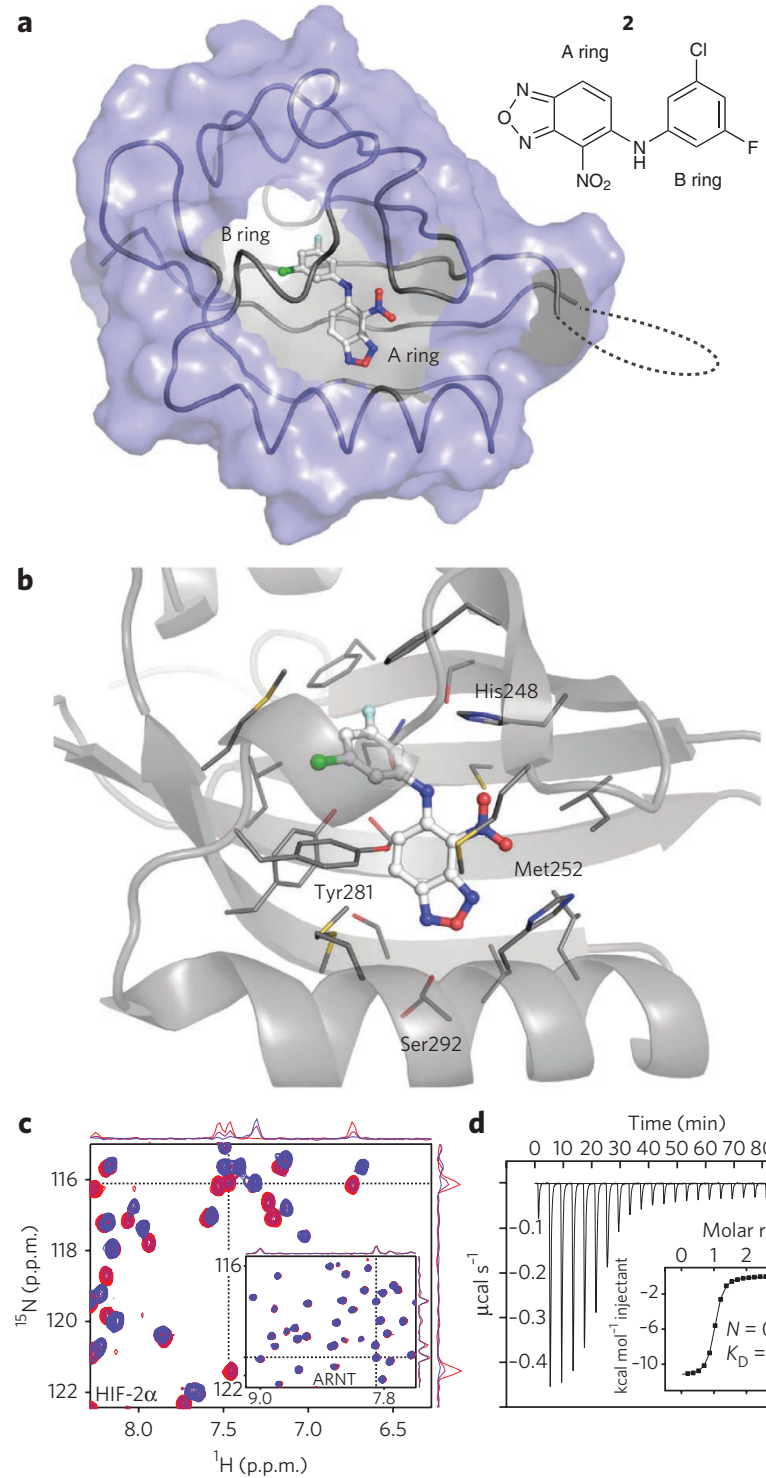

d Time $(\mathrm{min})$

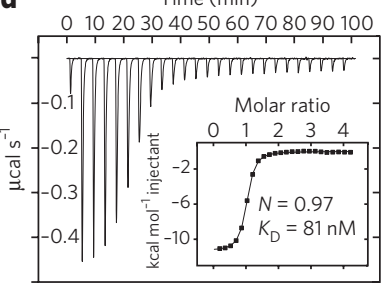

Figure 1 | Biophysical characterization of the HIF-2 $\alpha$ PAS-B-2 complex.

(a) The crystal structure of the ternary complex of HIF-2 PAS-B* with 2 reveals ligand binding into the internal cavity sequestered from bulk solvent within the HIF-2 $\alpha$ PAS-B domain (gray). For clarity, the ARNT-PAS-B* portion of the protein heterodimer is not shown, and a portion of the HIF- $2 \alpha$ PAS- $B^{\star}$ surface (blue) has been cut away to reveal the internal binding site. (b) Protein-ligand contacts as revealed by an expanded view of the binding site of $\mathbf{2}$, showing that it is composed of a mix of polar and hydrophobic residues. (c) ${ }^{15} \mathrm{~N}-{ }^{-1} \mathrm{H}$ HSQC spectra of $200 \mu \mathrm{M}\left[{ }^{15} \mathrm{~N}\right] \mathrm{HIF}-2 \alpha$ PAS-B (main panel) and [ $\left.{ }^{15} \mathrm{~N}\right] A R N T$ PAS-B (inset) in the presence of $0 \mu \mathrm{M}, 125 \mu \mathrm{M}$ and $250 \mu \mathrm{M} 2$ (red to blue) demonstrate the specific binding of 2 to HIF-2 $\alpha$ PAS-B. One-dimensional traces of spectra (at locations shown by dotted lines) demonstrate slow exchange binding behavior of $\mathbf{2}$ to HIF-2 $\alpha$ and no binding to ARNT PAS-B. (d) ITC measurements of 2 to HIF-2 $\alpha$ PAS-B quantify the binding affinity and 1:1 stoichiometry.

analog, 2, was identified (Fig. 1a) that also bound the HIF-2 $\alpha$ PAS-B domain (Fig. 1b-d) with a $K_{\mathrm{D}}$ of $81 \mathrm{nM}$ (Fig. 1d). Taken together, these data indicate that this class of compounds functions by binding HIF- $2 \alpha$ directly.

Antagonists bind within the HIF-2 $\alpha$ PAS-B internal cavity To further define how these compounds disrupt HIF-2 $\alpha$-ARNT PAS- $\mathrm{B}^{*}$ heterodimerization, we integrated X-ray crystallographic 

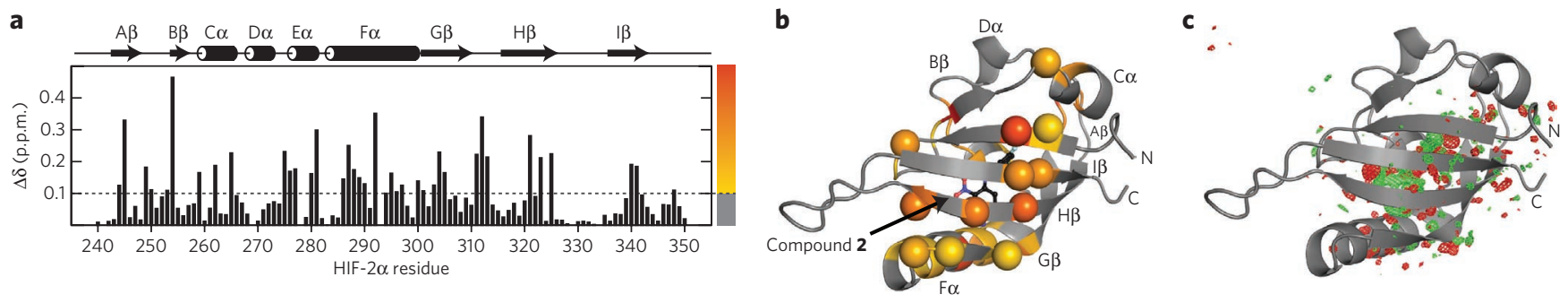

Figure 2 | Binding of 2 into HIF-2 $\boldsymbol{\alpha}$ PAS-B affects the heterodimeric $\boldsymbol{\beta}$-sheet interface between HIF PAS-B domains. (a) Backbone ${ }^{1} \mathrm{H}$ and ${ }^{15} \mathrm{~N}$ chemical shift differences between apo and 2-bound states are mapped onto the HIF-2 $\alpha$ PAS-B primary and secondary structures. (b) Ligand-induced chemical shift perturbations are mapped onto the HIF-2 $\alpha$ PAS-B structure with spheres denoting HIF- $2 \alpha C_{\alpha}$ sites within $8 \AA$ of ARNT PAS-B. View is approximately $180^{\circ}$ rotated about the $y$ (vertical) axis from the view in Figure 1a. The yellow-to-red color scale shown on the right of $\mathbf{a}$ is also used in $\mathbf{b}$ and Supplementary Figure 6. (c) Ligand-induced conformational changes in similar regions are also evident from X-ray diffraction data, as revealed by a $F_{0}$ (liganded) $F_{0}$ (apo) electron density difference map (rendered at $4 \sigma$; positive density in green, negative density in red).

and high-resolution solution NMR studies of HIF- $\alpha$ PAS-B bound to 2. As anticipated, a co-crystal structure revealed that this ligand bound within the preformed HIF- $2 \alpha$ PAS-B internal cavity (Fig. 1a,b, Supplementary Fig. 4 and Supplementary Table 2), displacing water. Compound 2 shares some common structural features with the previously described small-molecule ligands (Supplementary Fig. 5), facilitating binding with a combination of van der Waals and electrostatic interactions, including (i) the placement of the ligand nitro group and linker amine adjacent to the His 248 imidazole side chain, (ii) a $\pi$-hydrogen bond between the Tyr281 hydroxyl and the A-ring benzene ring and (iii) an intraligand hydrogen bond shared between the amine linker and the A ring nitro moiety. Further examination of the structure suggests that 2 derives its higher affinity from enhanced van der Waals and electrostatic interactions. In particular, the larger dihalogenated $\mathrm{B}$ ring of 2 better complements the surrounding hydrophobic pocket, which was only partially occupied by the B rings of the fragment-derived compounds THS-017, THS-020 and THS-044 that bound with dissociation constants ranging from $600 \mathrm{nM}$ to $2 \mu \mathrm{M}$ and lower molar enthalpies ${ }^{17,18}$ (Supplementary Fig. 5). In addition, the A ring of 2 presents potential new electrostatic interactions, particularly in its placement of the hydrogen-bonding oxadiazole ring adjacent to one of the Ser292 side chain conformations observed in the 2 ternary complex. Similarly, identification of B-ring substitutions that better complemented the apo protein binding site shape than those present in 1 was key to the development of 2 (Supplementary Fig. 5).

Bound ligands induce HIF-2 $\alpha$ PAS-B conformational changes To obtain mechanistic insights into the linkage between smallmolecule binding and protein-protein interactions in HIF- $2 \alpha$ PAS-B, we compared independent solution and crystallographic data acquired from apo and 2-bound HIF-2 $\alpha$. The HIF- $2 \alpha$ PASB-2 complex was amenable to high-resolution solution NMR studies (Fig. 1c), allowing us to assign backbone chemical shifts and compare these to existing apo protein information ${ }^{9}$. Chemical shift differences observed between these sets (Fig. 2a) established that 2 binding affected many sites in the surrounding protein, which still retains the mixed $\alpha-\beta$ PAS domain fold. Mapping these shift changes onto the crystal structure (Fig. 2a,b), we observed many of the largest differences on the $\beta$-sheet (particularly the $\mathrm{H} \beta$, I $\beta$ and $\mathrm{A} \beta$ strands). Parallel crystallographic analyses used a difference Fourier analysis of apo and 2-bound HIF-2 $\alpha$-ARNT. PAS-B $^{*}$ data sets (featuring highly similar crystal parameters) showed ligand-associated changes in electron density both within the HIF- $2 \alpha$ cavity and across the neighboring $\beta$-sheet (Fig. 2c). Notably, almost no $F_{\mathrm{o}}-F_{\mathrm{o}}$ difference density was observed elsewhere in the structure, including the ARNT PAS-B subunit, providing a powerful control for artifacts from the analysis.
These independent analyses link ligand binding to conformational changes at the $\beta$-sheet surface of the HIF- $2 \alpha$ PAS-B domain, which is used to bind its ARNT counterpart (Supplementary Fig. 6), strongly supporting an allosteric mode of action for our artificial inhibitors.

\section{Ligand 2 selectively disrupts HIF-2 heterodimerization}

Like 1 , the conformational changes induced by 2 disrupt heterodimerization of isolated HIF- $2 \alpha$ and ARNT PAS- ${ }^{*}$ domains in the AlphaScreen assay (Fig. 3a). However, heterodimerization of the two full-length HIF subunits is mediated by multiple protein-protein interactions involving the PAS-A and basic helix-loop-helix domains ${ }^{10}$. To determine whether 2 can antagonize heterodimerization between full-length HIF- $2 \alpha$ and ARNT polypeptides,

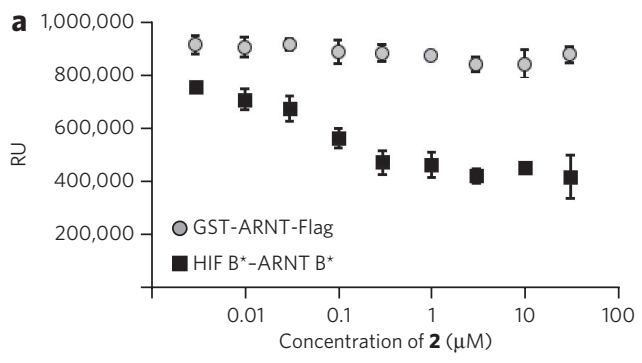

b

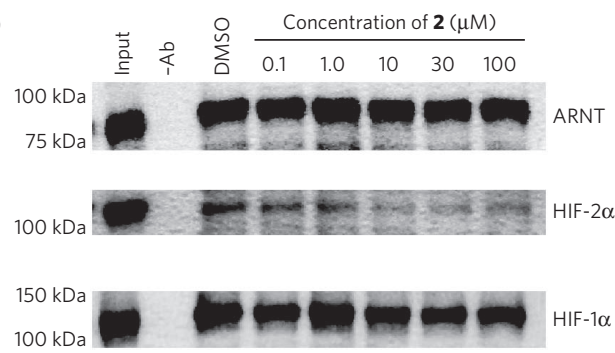

Figure 3 | 2 disrupts HIF-2 heterodimerization in vitro. (a) Addition of 2 blocks heterodimer assembly between purified recombinant HIF- $2 \alpha$ PAS- $B^{\star}$ and ARNT PAS- $B^{\star}$ heterodimer (squares) as assessed in the AlphaScreen assay. No effect was observed in control reactions using a single (doubly tagged) GST-ARNT-PAS-B*-Flag protein capable of recruiting both beads to induce an AlphaScreen signal (circles). Assays were performed in triplicate, and error bars represent \pm s.d. RU, relative units. (b) Compound $\mathbf{2}$ disrupts heterodimerization of the full-length HIF-2 transcription factor. Nuclear extracts prepared from hypoxic Hep3B cells expressing ARNT, HIF- $2 \alpha$ and HIF-1 $\alpha$ (input) were incubated with increasing concentrations of $\mathbf{2}$. Immunoblot analysis indicates amounts of HIF polypeptides immunoprecipitated in the absence (-Ab) or presence of an antibody to ARNT. 
a
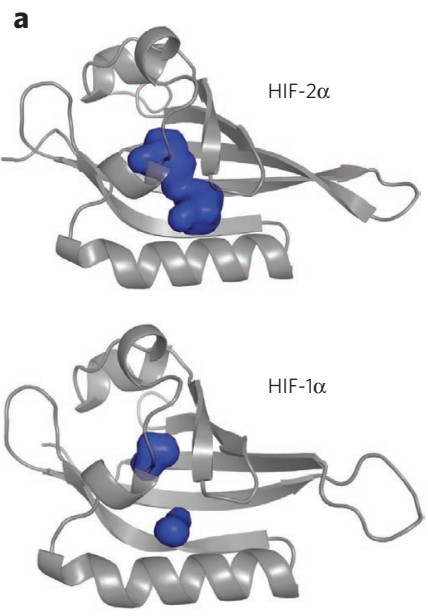

b.
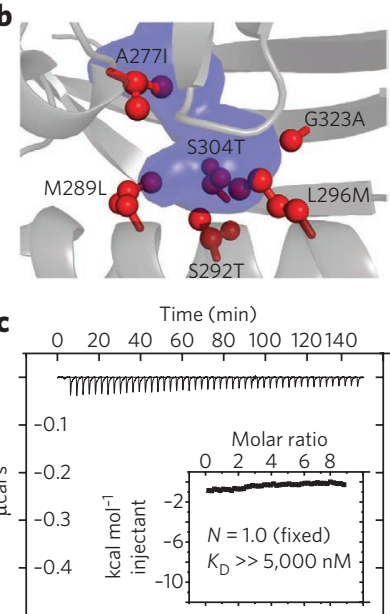

Figure 4 | 2 binds selectively to HIF- $2 \alpha$ over HIF-1 $\alpha$ PAS-B.

(a) Comparison of internal cavity sizes (blue) identified by a 1.4- $\AA$ probe within our HIF-2 $\alpha$ PAS-B crystal structure (PDB code 3F1P) ${ }^{18}$ (top) and a homology model of HIF-1 $\alpha$ PAS-B domain based on this structure. Sequence differences among these two closely related paralogs reduce the expected size of the HIF-1 $\alpha$ PAS-B cavity. (b) The HIF-1 $\alpha$ PAS-B model suggests that several sequence differences among these paralogs lead to the placement of bulkier side chains (red) within the HIF-1 $\alpha$ PAS-B core. These substitutions seem to shrink the cavity observed in HIF-2 $\alpha$ PAS-B (HIF-2 $\alpha$ PAS-B cavity rendered as a blue surface, superimposed on the HIF-1 $\alpha$ PAS-B model). Amino acid differences are indicated with the first letter designating the HIF-2 amino acid identity and the last letter designating the HIF-1 identity. (c) ITC measurements of a HIF-1 $\alpha$ PAS-B-2 titration does not show detectable protein-ligand interaction under the same conditions used to observe binding with HIF- $2 \alpha$ PAS-B (Fig. 1d).

we prepared nuclear extracts from hypoxic Hep3B cells. An antibody recognizing the $\mathrm{N}$-terminus of $\mathrm{ARNT}^{10}$ was used to immunoprecipitate the endogenous ARNT protein from the nuclear extracts (Fig. 3b and Supplementary Fig. 7). The HIF-2 $\alpha$ subunit coimmunoprecipitated with ARNT in extracts incubated with the DMSO vehicle control. However, addition of 2 to the extracts decreased HIF- $2 \alpha$ coimmunoprecipitation efficiency by more than a factor of two in a dose-dependent manner (Fig. 3b). The magnitude of these effects on HIF-2 heterodimerization is similar to that observed following mutation of the HIF-2 $\alpha$ PAS-B dimerization interface ${ }^{10}$.

In addition to HIF- $2 \alpha$, Hep3B cells also express HIF-1 $\alpha$. Though these two HIF- $\alpha$ isoforms share $>70 \%$ identity between their PAS-B domains, modeling of the HIF- $1 \alpha$ PAS-B domain onto the HIF- $2 \alpha$ PAS-B structure suggests that several bulkier residues face into the internal HIF-1 $\alpha$ cavity (Fig. 4a,b and Supplementary Fig. 8). Such alterations are expected to constrict the pocket and interfere with ligand binding, and this was confirmed by ITC data demonstrating that 2 effectively does not bind the HIF-1 $\alpha$ PAS-B domain (Fig. 4c; $\left.K_{\mathrm{D}} \gg>5 \mathrm{M}\right)$. The large selectivity of 2 for HIF- $2 \alpha$ is shown in Figure $3 \mathbf{b}$, as increasing amounts of 2 have little effect on full-length HIF-1 heterodimerization as assessed by coimmunoprecipitation. These data confirm that, in vitro, 2 binds selectively within a preformed ligand binding site buried within the HIF- $2 \alpha$ PAS-B domain. Ensuing allosteric conformational changes propagate to the surface of the domain, weakening interactions with the ARNT PAS-B domain and disrupting heterodimerization of the full-length HIF-2 transcription factor.

\section{Ligand 2 selectively disrupts HIF-2 in cultured cells}

The improved in vitro efficacy of 2 provides an opportunity to validate selective HIF-2 antagonism in living cells. No overt toxicity was observed for 786-0 or Hep3B cells incubated with as much as $30 \mu \mathrm{M}$ of 2 (Supplementary Fig. 9). Compound 2 was not readily metabolized as shown by a $\sim 14$-h half-life $\left(t_{1 / 2}\right)$ when incubated with 786-0 cells in vitro; parallel experiments using only culture medium showed no loss over $24 \mathrm{~h}$ (data not shown). The 786- 0 cells derived from a human renal cell carcinoma lack a functional pVHL, the E3 ubiquitin ligase responsible for marking HIF- $\alpha$ for proteasomal degradation under normoxic conditions. These cells also lack detectable HIF-1 $\alpha$ expression so that HIF-dependent regulation of target genes is attributable to the HIF-2 isoform that constitutively accumulates ${ }^{25}$. Addition of 2 to cultured 786-0 cells does not alter HIF-2 $\alpha$ expression at either the mRNA (Fig. 5a) or protein level (Supplementary Fig. 10). However, expression of a well-validated HIF-2 target gene (VEGFA) is reduced in a dose-dependent manner in 786- 0 cells incubated with 2 for 18 h (Fig. 5a).

To confirm that the mode of action for HIF-2 inhibition by 2 is indeed dependent upon binding to HIF-2 $\alpha$, we examined ligand effects on Hep3B cells. Although some hypoxia-inducible target genes are regulated by both HIF-1 and HIF-2 in these cells, other genes are exclusively regulated by a single isoform ${ }^{26,27}$. To examine EPO or PGK1 expression as surrogate markers for HIF-2 and HIF-1, respectively, Hep3B cells were preincubated with $1 \mu \mathrm{M}$ or $10 \mu \mathrm{M} 2$ for $2 \mathrm{~h}$ and maintained either under normoxic or under hypoxic $\left(1 \% \mathrm{O}_{2}\right)$ conditions for $6 \mathrm{~h}$ or $12 \mathrm{~h}$. Whereas hypoxia induces both EPO and PGK1 mRNA expression, only hypoxic induction of EPO mRNA is antagonized by 2 (Fig. 5b). Incubation with 2 has no effect a

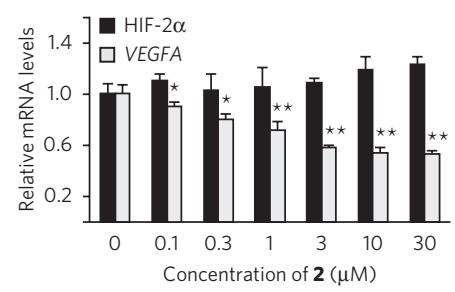

b

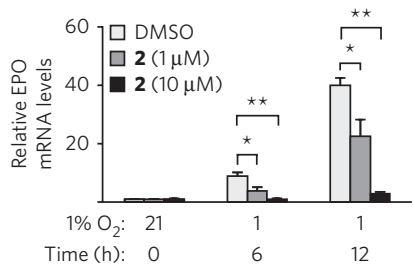

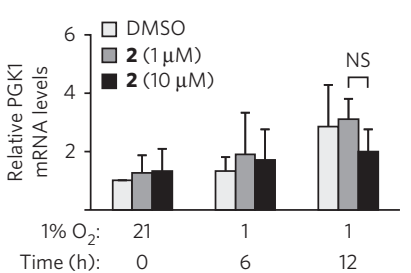

Figure $\mathbf{5}$ | 2 selectively antagonizes HIF-2 activity in cultured cells. (a,b) Although incubation of $\mathbf{2}$ with normoxic $786-0$ cells has no effect on HIF-2 $\alpha$ expression (a), RT-PCR reveals that $\mathbf{2}$ antagonizes expression of the HIF-2 target genes VEGFA in 786-0 cells (a) and EPO in Hep3B (b, left) cells. In contrast, transcription of a HIF-1-selective target gene (PGK1) in Hep3B cells is unaffected by $\mathbf{2}$ (b, right). (c) Compound $\mathbf{2}$ selectively disrupts DNA binding by HIF-2, but not HIF-1, in a ChIP assay. The RT-PCR data for each gene are the mean of three values determined from three independently harvested sets, and the error bars represent \pm s.d. Differences between paired values are statistically significant as determined by Student's $t$-test. ${ }^{\star} P<0.01 ;{ }^{\star \star} P<0.001 ; \mathrm{NS}$, nonsignificant. 
on the expression of PGK1 or on the HIF- $1 \alpha$ and HIF- $2 \alpha$ mRNA levels (Supplementary Fig. 11).

If 2 is working in cells by antagonizing HIF-2 heterodimerization, HIF-2's DNA-binding activity should likewise be selectively compromised. Chromatin immunoprecipitation (ChIP) using antibodies raised against HIF- $1 \alpha$ or HIF- $2 \alpha$ was used to measure HIF DNA binding in cultured cells. An increase in both HIF-1 and HIF-2 binding to a HIF-responsive promoter element is observed under hypoxic conditions, reflecting the increase in stability of both $\alpha$-subunits. However, the DNA-binding activity of HIF-1 is unaffected in cells incubated with $10 \mu \mathrm{M} 2$, whereas HIF-2's DNAbinding activity is substantially decreased (Fig. 5c). Together these data constitute a proof-of-principle demonstration that smallmolecule ligands can directly and selectively bind a cavity within the PAS-B domain of the HIF- $2 \alpha$ polypeptide. Ligand binding induces conformational changes in HIF- $2 \alpha$ that disrupt formation of the HIF-2 heterodimer, antagonizing HIF-2's DNA-binding activity and selectivity reducing expression of HIF-2 target genes in living cells.

\section{DISCUSSION}

Great progress has been made in unraveling the relationship between HIF, hypoxia and tumor progression and metastasis (reviewed in refs. 19,28). Increased amounts of HIF have been observed in human cancers of the brain, breast, kidney and ovaries, among others, and are often associated with increased tumor aggressiveness, therapeutic resistance and mortality ${ }^{28}$. Although much attention has focused on links between HIF- $1 \alpha$ and cancer, there is increasing evidence that HIF- $2 \alpha$ is an important driver of a number of common tumors ${ }^{19,29}$. For example, a clear role for HIF-2 in cancer has been defined for a number of VHL-deficient renal cell carcinomas in which the protumorigenic effects of stabilized HIF- $2 \alpha$ cannot be phenocopied by HIF-1 $\alpha^{6-8}$. More recently, HIF- $2 \alpha$ has been recognized as a notable therapeutic target in a number of genetically diverse cancers ${ }^{30}$, including glioblastomas ${ }^{31,32}$ and non-small-cell lung carcinomas ${ }^{33}$. The underlying mechanisms responsible for a HIF-2 preference in certain cancers remain the subject of investigation, although selectivity in expression, target genes and interaction partners have all been implicated ${ }^{19}$.

The accumulation and activity of the HIF- $\alpha$ subunit is acutely induced following a decrease in cellular $\mathrm{O}_{2}$, as is frequently encountered in tumors. Under normoxia, the $\alpha$-subunit is rapidly degraded following $\mathrm{O}_{2}$-dependent hydroxylation of key prolines within the oxygen-dependent degradation domain, recruiting the pVHL ubiquitin ligase ${ }^{34,35}$. Parallel $\mathrm{O}_{2}$-dependent hydroxylation of asparagines in the HIF- $\alpha$ C-terminal transactivation domain also controls HIF's ability to interact with certain transcriptional coactivators $^{36,37}$. However, HIF- $\alpha$ is often constitutively upregulated in an $\mathrm{O}_{2}$-independent manner in tumors containing appropriate genetic alterations to oncogenic signaling pathways or tumor suppressor genes. This scenario is best exemplified by mutations that inactivate $\mathrm{pVHL}$ and result in constitutive stabilization of the HIF- $\alpha$ subunit, predisposing patients to renal cell carcinomas and a host of other cancers ${ }^{38}$. Mutations to many other proteins have also been shown to constitutively induce HIF- $\alpha$, albeit by other mechanisms ${ }^{28}$. Small-molecule HIF antagonists that directly bind the transcription factor, independent of the mechanism underlying its induction, may therefore have utility in a wide range of disease studies.

HIF- $2 \alpha$ provides a particularly appealing target for inhibitor development given the preformed cavity present in its PAS-B domain. Such cavities are very rare in protein domains of this size, as they compromise a major component of the hydrophobic core that normally stabilizes a protein fold. Despite its sequestration from solvent, we have previously shown that this cavity is accessible to small-molecule ligands in vitro ${ }^{17,18}$. Here we identify superior compounds that bind the HIF- $2 \alpha$ PAS-B domain with a $K_{\mathrm{D}} \sim 80 \mathrm{nM}$. This class of compounds induces conformational changes within the domain upon binding, with changes identified at the critical $\beta$-sheet interface used to bind ARNT PAS-B (Supplementary Fig. 12). Notably, comparable changes are observed in PAS domains with their cognate natural ligands despite differences in ligand structure and activation mechanism, suggesting some degree of conservation in their allosteric activation principles. For example, photosensory PAS domains, which bind flavin chromophores comparably to our artificial HIF- $2 \alpha$ ligands, harness a change in cofactor configuration via the photochemical formation of a protein-flavin bond to perturb $\beta$-sheet structure and protein binding ${ }^{14,39}$ (Supplementary Fig. 12).

Though multiple protein-protein interactions drive HIF heterodimerization, ligand-dependent allosteric changes to the HIF- $2 \alpha$ PAS-B domain are sufficient to disrupt formation of the endogenous full-length HIF-2 complex, with a corresponding selective diminution of HIF-2 activity in living cells. Though HIF PAS domains have been implicated as targets for HIF inihibitors ${ }^{40,41}$, this is to our knowledge the first example in which the molecular underpinnings for compound activity have been elucidated. For example, acriflavine was recently reported to directly antagonize HIF upon binding to the HIF- $\alpha$ PAS-B domains ${ }^{41}$. However, as this compound inhibits both HIF-1 and HIF-2, an internal PAS-B cavity is most likely not the relevant binding site as differences among the isoforms confer exquisite specificity.

Though initially characterized in the context of their role in oxygen sensing, HIFs are responsive to a multitude of cellular cues, including cellular metabolites. For example, the nutrient-sensing mTOR pathway acts upstream of HIF- $\alpha$ expression, whereas intermediary metabolites of the Krebs cycle influence the activity of the HIF hydroxylases ${ }^{42}$. Although our findings here provide useful reagents for HIF-2 inhibition, it is tempting to further speculate that such a pocket has evolved for HIF-2 regulation by endogenous metabolites in vivo. From a structural perspective, this hypothesis is supported by the presence of rare large cavities (such as in HIF- $2 \alpha$ PAS-B) most commonly within apo forms of natural ligand-binding proteins (Supplementary Fig. 1). If so, future elucidation of cognate natural ligands may provide new insights into isoform-specific metabolic regulation of HIF in physiological and pathological settings.

Received 13 August 2012; accepted 18 January 2013; published online 24 February 2013

\section{METHODS}

Methods and any associated references are available in the online version of the paper.

Accession codes. Protein Data Bank (PDB): The crystallographic data for HIF- $2 \alpha-$ ARNT PAS- $^{*}-2$ is deposited under accession code $4 \mathrm{GHI}$.

\section{References}

1. Semenza, G.L. Hypoxia-inducible factors: mediators of cancer progression and targets for cancer therapy. Trends Pharmacol. Sci. 33, 207-214 (2012).

2. Xia, X. et al. Integrative analysis of HIF binding and transactivation reveals its role in maintaining histone methylation homeostasis. Proc. Natl. Acad. Sci. USA 106, 4260-4265 (2009).

3. Schödel, J. et al. High-resolution genome-wide mapping of HIF-binding sites by ChIP-seq. Blood 117, e207-e217 (2011).

4. Majmundar, A.J., Wong, W.J. \& Simon, M.C. Hypoxia-inducible factors and the response to hypoxic stress. Mol. Cell 40, 294-309 (2010).

5. Greer, S.N., Metcalf, J.L., Wang, Y. \& Ohh, M. The updated biology of hypoxia-inducible factor. EMBO J. 31, 2448-2460 (2012).

6. Kondo, K., Kim, W.Y., Lechpammer, M. \& Kaelin, W.G. Jr. Inhibition of HIF $2 \alpha$ is sufficient to suppress pVHL-defective tumor growth. PLoS Biol. 1, E83 (2003).

7. Kondo, K., Klco, J., Nakamura, E., Lechpammer, M. \& Kaelin, W.G. Jr. Inhibition of HIF is necessary for tumor suppression by the von HippelLindau protein. Cancer Cell 1, 237-246 (2002).

8. Maranchie, J.K. et al. The contribution of VHL substrate binding and HIF1- $\alpha$ to the phenotype of VHL loss in renal cell carcinoma. Cancer Cell 1, 247-255 (2002) 
9. Erbel, P.J., Card, P.B., Karakuzu, O., Bruick, R.K. \& Gardner, K.H. Structural basis for PAS domain heterodimerization in the basic helix-loop-helix-PAS transcription factor hypoxia-inducible factor. Proc. Natl. Acad. Sci. USA 100, 15504-15509 (2003).

10. Yang, J. et al. Functions of the Per/ARNT/Sim (PAS) domains of the hypoxia inducible factor (HIF). J. Biol. Chem. 280, 36047-36054 (2005)

11. Partch, C.L., Card, P.B., Amezcua, C.A. \& Gardner, K.H. Molecular basis of coiled coil coactivator recruitment by the aryl hydrocarbon receptor nuclear translocator (ARNT). J. Biol. Chem. 284, 15184-15192 (2009).

12. Partch, C.L. \& Gardner, K.H. Coactivators necessary for transcriptional output of the hypoxia inducible factor, HIF, are directly recruited by ARNT PAS-B. Proc. Natl. Acad. Sci. USA 108, 7739-7744 (2011).

13. Henry, J.T. \& Crosson, S. Ligand-binding PAS domains in a genomic, cellular, and structural context. Annu. Rev. Microbiol. 65, 261-286 (2011).

14. Harper, S.M., Neil, L.C. \& Gardner, K.H. Structural basis of a phototropin light switch. Science 301, 1541-1544 (2003).

15. Wells, J.A. \& McClendon, C.L. Reaching for high-hanging fruit in drug discovery at protein-protein interfaces. Nature 450, 1001-1009 (2007).

16. Koehler, A.N. A complex task? Direct modulation of transcription factors with small molecules. Curr. Opin. Chem. Biol. 14, 331-340 (2010).

17. Key, J., Scheuermann, T.H., Anderson, P.C., Daggett, V. \& Gardner, K.H. Principles of ligand binding within a completely buried cavity in HIF2 $\alpha$ PAS-B. J. Am. Chem. Soc. 131, 17647-17654 (2009).

18. Scheuermann, T.H. et al. Artificial ligand binding within the HIF2 $\alpha$ PAS-B domain of the HIF2 transcription factor. Proc. Natl. Acad. Sci. USA 106, 450-455 (2009).

19. Qing, G. \& Simon, M.C. Hypoxia inducible factor-2 $\alpha$ : a critical mediator of aggressive tumor phenotypes. Curr. Opin. Genet. Dev. 19, 60-66 (2009).

20. Morris, M.R. et al. Mutation analysis of hypoxia-inducible factors HIF1 $\alpha$ and HIF2 $\alpha$ in renal cell carcinoma. Anticancer Res. 29, 4337-4343 (2009).

21. Kaelin, W.G. Jr. Treatment of kidney cancer: insights provided by the VHL tumor-suppressor protein. Cancer 115, 2262-2272 (2009).

22. Shen, C. et al. Genetic and functional studies implicate HIF1 $\alpha$ as a 14q kidney cancer suppressor gene. Cancer Discov. 1, 222-235 (2011).

23. Amezcua, C.A., Harper, S.M., Rutter, J. \& Gardner, K.H. Structure and interactions of PAS kinase N-terminal PAS domain: model for intramolecular kinase regulation. Structure 10, 1349-1361 (2002).

24. Rogers, J.L. et al. Development of inhibitors of the PAS-B domain of the HIF-2 $\alpha$ transcription factor. J. Med. Chem. 10.1021/jm301847z (30 January 2013).

25. Krieg, M. et al. Up-regulation of hypoxia-inducible factors HIF-1 $\alpha$ and HIF- $2 \alpha$ under normoxic conditions in renal carcinoma cells by von Hippel-Lindau tumor suppressor gene loss of function. Oncogene 19, 5435-5443 (2000).

26. Dioum, E.M. et al. Regulation of hypoxia-inducible factor $2 \alpha$ signaling by the stress-responsive deacetylase sirtuin 1. Science 324, 1289-1293 (2009).

27. Scortegagna, M. et al. HIF- $2 \alpha$ regulates murine hematopoietic development in an erythropoietin-dependent manner. Blood 105, 3133-3140 (2005).

28. Semenza, G.L. Targeting HIF-1 for cancer therapy. Nat. Rev. Cancer 3, 721-732 (2003).

29. Keith, B., Johnson, R.S. \& Simon, M.C. HIF $1 \alpha$ and HIF2 $\alpha$ : sibling rivalry in hypoxic tumour growth and progression. Nat. Rev. Cancer 12, 9-22 (2012).

30. Franovic, A., Holterman, C.E., Payette, J. \& Lee, S. Human cancers converge at the HIF-2 $\alpha$ oncogenic axis. Proc. Natl. Acad. Sci. USA 106, 21306-21311 (2009).

31. Holmquist-Mengelbier, L. et al. Recruitment of HIF- $1 \alpha$ and HIF- $2 \alpha$ to common target genes is differentially regulated in neuroblastoma: HIF- $2 \alpha$ promotes an aggressive phenotype. Cancer Cell 10, 413-423 (2006)

32. Pietras, A. et al. HIF- $2 \alpha$ maintains an undifferentiated state in neural crest-like human neuroblastoma tumor-initiating cells. Proc. Natl. Acad. Sci. USA 106, 16805-16810 (2009).
33. Mazumdar, J. et al. HIF-2 $\alpha$ deletion promotes Kras-driven lung tumor development. Proc. Natl. Acad. Sci. USA 107, 14182-14187 (2010).

34. Ivan, M. et al. HIF $\alpha$ targeted for VHL-mediated destruction by proline hydroxylation: implications for $\mathrm{O}_{2}$ sensing. Science 292, 464-468 (2001).

35. Jaakkola, P. et al. Targeting of HIF- $\alpha$ to the von Hippel-Lindau ubiquitylation complex by $\mathrm{O}_{2}$-regulated prolyl hydroxylation. Science 292, 468-472 (2001).

36. Lando, D. et al. FIH-1 is an asparaginyl hydroxylase enzyme that regulates the transcriptional activity of hypoxia-inducible factor. Genes Dev. 16, 1466-1471 (2002).

37. Lando, D., Peet, D.J., Whelan, D.A., Gorman, J.J. \& Whitelaw, M.L. Asparagine hydroxylation of the HIF transactivation domain: a hypoxic switch. Science 295, 858-861 (2002).

38. Kaelin, W.G. Von Hippel-Lindau disease. Annu. Rev. Pathol. 2, 145-173 (2007).

39. Halavaty, A.S. \& Moffat, K. N- and C-terminal flanking regions modulate light-induced signal transduction in the LOV2 domain of the blue light sensor phototropin 1 from Avena sativa. Biochemistry 46, 14001-14009 (2007).

40. Park, E.J. et al. Targeting the PAS-A domain of HIF-1 $\alpha$ for development of small molecule inhibitors of HIF-1. Cell Cycle 5, 1847-1853 (2006).

41. Lee, K. et al. Acriflavine inhibits HIF-1 dimerization, tumor growth, and vascularization. Proc. Natl. Acad. Sci. USA 106, 17910-17915 (2009).

42. Semenza, G.L. HIF-1: upstream and downstream of cancer metabolism. Curr. Opin. Genet. Dev. 20, 51-56 (2010).

\section{Acknowledgments}

The authors thank S. Wang and members of the University of Texas Southwestern High-Throughput Screening Core Facility, D. Tomchick, C. Brautigam, J. MacMillan, N. Williams and members of our laboratories for their help. This work was funded by grants from the US National Institutes of Health (P01 CA095471, P30 CA142543) and the Cancer Prevention Research Institute of Texas (RP-100846). R.K.B. is the Michael L. Rosenberg Scholar in Medical Research and was supported by a Career Award in the Biomedical Sciences from the Burroughs Wellcome Fund; K.H.G. is the Virginia Lazenby O'Hara Chair in Biochemistry and W.W. Caruth Scholar in Biomedical Research; and U.K.T. is a W.W. Caruth Jr. Scholar in Biomedical Research. J.A.G. was supported by funds provided by the Department of Veterans Affairs. Results shown in this report are derived from work performed at Argonne National Laboratory, Structural Biology Center at the Advanced Photon Source. Argonne National Laboratory is operated by UChicago Argonne, LLC, for the US Department of Energy, Office of Biological and Environmental Research under contract DE-AC02-06CH11357. This investigation was conducted in a facility constructed with support from the Research Facilities Improvement Program (grant no. C06 RR 15437-01) from the National Center for Research Resources, US National Institutes of Health.

\section{Author contributions}

R.K.B. and K.H.G. conceived and designed the experiments. T.H.S., Q.L., H.-W.M., L.Z., R.C., J.N. and J.L. performed the experiments. R.K.B., K.H.G., T.H.S., J.K., J.A.G., D.E.F. and U.K.T. analyzed the data. R.K.B., K.H.G. and T.H.S. wrote the paper.

\section{Competing financial interests}

The authors declare competing financial interests: details accompany the online version of the paper.

\section{Additional information}

Supplementary information, chemical compound information and chemical probe information is available in the online version of the paper. Reprints and permissions information is available online at http://www.nature.com/reprints/index.html. Correspondence and requests for materials should be addressed to R.K.B. 


\section{ONLINE METHODS}

Protein preparation. HIF- $2 \alpha$ PAS-B (240-350), HIF-2 $\alpha$ PAS-B ${ }^{*}(240-350$, R247E), ARNT PAS-B (355-470) and ARNT PAS-B* (355-470, E362R) domains were expressed and purified as previously described ${ }^{18}$. HIF- $1 \alpha$ PAS-B (238-349) used for ITC and HIF-2 $\alpha$ PAS-B used for NMR studies complexed with 2 were expressed with an $\mathrm{N}$-terminal G $\beta 1$ fusion $\operatorname{tag}^{23}$ and purified by Source-Q ion exchange and Superdex S75 size exclusion chromatography. For the HIF- $1 \alpha$ PAS-B domain, the G $\beta 1$ tag was not removed, as its retention improved the solubility of the purified PAS-B domain.

AlphaScreen protein reagents were expressed as GST-HIF- $2 \alpha$ PAS- $^{*}$ and His $_{6}$-G $\beta 1$-ARNT-PAS-B*-Flag fusions, purified with affinity (glutathione or $\mathrm{Ni}(\mathrm{II})$ ) and Superdex $\mathrm{S} 75$ chromatography, equilibrated in AlphaScreen assay buffer (50 mM Tris ( $\mathrm{pH} 7.5), 100 \mathrm{mM} \mathrm{NaCl} ; 1 \mathrm{mM}$ dithiothreitol) and flash-frozen in liquid $\mathrm{N}_{2}$.

NMR analyses. Protein backbone resonance assignments for the HIF- $2 \alpha$ PAS$\mathrm{B}-2$ complex were determined using $\mathrm{HNCO}$, HNCACB and $\mathrm{CBCA}(\mathrm{CO}) \mathrm{NH}$ spectra collected on a cryoprobe-equipped Varian Inova $600 \mathrm{MHz}$ spectrometer from a sample of $300 \mu \mathrm{M} \mathrm{U}-\left[{ }^{13} \mathrm{C},{ }^{15} \mathrm{~N}\right] \mathrm{HIF}-2 \alpha \mathrm{PAS}-\mathrm{B}, 350 \mu \mathrm{M} 2$ and $0.4 \%$ DMSO in $10 \mathrm{mM} \mathrm{d}_{11}$-Tris, $\mathrm{pH} 7.3$, and $20 \mathrm{mM} \mathrm{NaCl}$ buffer using NMRView ${ }^{43}$. Data collected at a second condition (5 mM MES, pH $6.5 ; 20 \mathrm{mM} \mathrm{NaCl})$ were used to resolve ambiguities stemming from exchange broadening in a limi ted number of sites. Chemical shift differences (Fig. 2a,b) were calculated from backbone HIF-2 $\alpha$ PAS-B ${ }^{15} \mathrm{~N}-{ }^{1} \mathrm{H}$ assignments of the complex (here) and apo form ${ }^{9}$

$$
\Delta \delta=\sqrt{\left(\Delta \delta^{1} H\right)^{2}+\left(0.1 * \Delta \delta^{15} N\right)^{2}}
$$

AlphaScreen. Reactions were performed in microtiter plates containing $100 \mathrm{nM}$ GST-HIF-2 $\alpha$ PAS-B ${ }^{*}, 100 \mathrm{nM}$ ARNT-PAS-B ${ }^{*}$-Flag, $20 \mathrm{mM}$ Tris$\mathrm{Cl}$ (pH 7.5), $100 \mathrm{mM} \mathrm{NaCl}, 1 \mathrm{mM} \mathrm{DTT}, 0.02 \%$ Tween-20, $1.5 \mathrm{pg} / \mu 1$ AlphaScreen Glutathione Donor Beads (PerkinElmer) and $1.5 \mathrm{pg} / \mu \mathrm{l}$ AlphaLISA anti-Flag Acceptor Beads (PerkinElmer). Compound stocks (100x) were prepared in DMSO. Plates were incubated in the dark at room temperature with gentle rocking for $4 \mathrm{~h}$ before data collection using an EnVision microplate reader (PerkinElmer). As a control, the individual PAS-B ${ }^{\star}$ domains were replaced by a single (doubly tagged) GST-ARNT-PAS-B ${ }^{*}$-Flag protein $(230 \mathrm{nM})$ capable of recruiting both beads to induce an AlphaScreen signal.

NMR ligand binding assay. Compounds were titrated at $125-\mu \mathrm{M}$ and $250-\mu \mathrm{M}$ concentrations into samples of $200 \mu \mathrm{M}$ uniformly ${ }^{15} \mathrm{~N}$-labeled HIF- $2 \alpha$ and ARNT PAS-B domains. Changes in peak intensity or locations in ${ }^{15} \mathrm{~N}-{ }^{1} \mathrm{H}$ HSQC spectra indicated ligand binding.

Isothermal titration calorimetry of protein-small molecule complexes. Thermodynamic parameters of small-molecule binding were determined using a MicroCal VP-ITC calorimeter. Protein solutions were extensively dialyzed against buffer (50 mM Tris ( $\mathrm{pH} 7.5$ ), $20 \mathrm{mM} \mathrm{NaCl}$ and $5 \mathrm{mM}$ $\beta$-mercaptoethanol), which was subsequently used to prepare a matched compound solution by dilution from a $50-\mathrm{mM}$ compound stock in $100 \%$ DMSO. ITC data collected for 1 were acquired in 5.0\% DMSO to improve compound solubility, and data for 2 was collected at $0.02 \%$ DMSO. Prior controls have demonstrated modest effects of $5 \%$ DMSO on measured thermodynamic parameters for HIF-ligand complexes, typically reducing affinities by a factor of two to four ${ }^{17}$. Each isotherm was recorded by injecting $200 \mu \mathrm{M}$ protein (syringe) into 5- to $10-\mu \mathrm{M}$ solutions of compound (cell), accounting for dilution heats by subtracting data from a control titration of $200 \mu \mathrm{M}$ protein into a matched buffer-DMSO solution. Thermograms were fit to a single-site binding model to extract equilibrium binding parameters.

Crystallography. Compound 2 was co-crystallized as a ternary complex with the HIF- $2 \alpha-$ ARNT PAS- $B^{*}$ heterodimer ${ }^{17,18}$. Briefly, HIF- $2 \alpha-$ ARNT PAS- ${ }^{*}$ heterodimers were crystallized in the presence of a stoichiometric excess of 2. Ternary complex crystals grew in hanging drops of $2 \mu \mathrm{l}$ of $300 \mu \mathrm{M}$ ternary complex and $2 \mu \mathrm{l}$ of precipitant (100 mM Bis-Tris (pH 5.5-6.0), $20 \mathrm{mM} \mathrm{NaCl}$, 19-23\% PEG 3350), which was supplemented with 25\% PEG400 before freezing in liquid nitrogen. X-ray diffraction data were collected at the Advanced Photon Source (Argonne National Laboratory, Argonne, IL), beamline ID-19 at $100 \mathrm{~K}$ using $0.97937-\AA \mathrm{X}$-rays, which were reduced and scaled with the
HKL2000 software package ${ }^{44}$. The structures were determined, refined and validated using the PHENIX ${ }^{45}$ macromolecular crystallography software suite (version 1.7.2-869) in conjunction with the PRODRG2 web server ${ }^{46}$ to generate initial ligand coordinates, molecular modeling with $\mathrm{COOT}^{47}$, validation with MolProbity ${ }^{48}$, and additional analysis and figure preparation in PyMOL (Schrödinger, Inc.). The final structural model was refined with an occupancy of 2 at 0.7 , with final refinement statistics are presented in Supplementary Table 2 (coordinates deposited at RCSB with PDB code 4GHI). Calculated hydrogen atomic positions were added to protein and ligand coordinate files and were used in a 'riding-hydrogen' mode. The final model demonstrates good stereochemical properties, as accessed by Ramachandran (100\% favored) and MolProbity (3.17 (98\%) clash score and 1.24 (96\%) MolProbity scores) analyses. An $F_{\mathrm{o}}-F_{\mathrm{o}}$ electron density difference map (Fig. $2 \mathrm{c}$ and Supplementary Fig. 12a) was calculated using difference structure factor amplitudes derived from the apo and ligand-bound diffraction data, with phases derived from the atomic coordinates of the apo protein heterodimer (PDB code $3 \mathrm{~F} 1 \mathrm{P}^{18}$ ). Structure factor amplitudes were scaled using SCALEIT, and maps were calculated using FFT, both from the CCP4 (ref. 49) software suite. The homology model of HIF- $1 \alpha$ PAS-B (Fig. $4 \mathbf{a}, \mathbf{b}$ ) was generated with MODELLER ${ }^{50}$ using the ligand-free coordinates of HIF-2 $\alpha$ PAS-B (PDB code 3F1P).

Occupancies for sites demonstrating multiple conformers were optimized by phenix.refine including the HIF-2 $\alpha$ PAS-B cavity-lining residue Met252. As 2 demonstrates fractional (0.7) occupancy of binding sites in the crystal, both apo (Met252-in) and 2-bound (Met252-out) conformations are represented in $2 \mathrm{~m} F_{\mathrm{o}}-\mathrm{D} F_{\mathrm{c}}$ density maps (Supplementary Fig. 4). The Met252-in conformation is not modeled in the final structure for clarity. Fractional occupancy of 2 is most likely a consequence of steric constraints imposed by growing HIF- $2 \alpha$ PAS- $B^{*}-2$ crystals under the same conditions previously used grow apo heterodimer crystals. This approach might constrain compound-induced protein conformational changes associated with PAS- $\mathrm{B}^{\star}$ heterodimer disruption, reducing HIF- $2 \alpha$ PAS- ${ }^{\star}$-compound affinities and ligand occupancies in the ternary complex.

Buried cavity identification. Solvent-inaccessible cavities were detected, and volumes were quantified using an in-house Python-based software program developed specifically for locating internal, buried cavities (available upon request). The program uses a grid-based search to identify regions within the solvent-accessible molecular envelope where a 1.4- $\AA$ probe sphere, which approximates a water molecule, may be placed without sterically clashing with protein. Adjacent probe-occupied points were iteratively clustered into groups with successively finer grid spacing. At each stage, clusters that extend to the solvent are discarded. Finally, volumes of the contiguous clusters that remain were determined. This approach was applied to a nonredundant subset of the PDB generated on the basis of sequence homology between X-ray crystal structures of $2.5 \AA$ or better resolution (generated by the NCBI VAST tool, nonidentical data set $=32,263$ chains as of February 2012). Cavities containing nonwater HETATM records, commonly used for ligands and cofactors, were excluded from analysis. This analysis revealed 121,433 cavities with a mean volume of $38.9 \AA^{3}$.

Cell culture. Human renal adenocarcinoma 786-0 and hepatocellular carcinoma Hep3B cells (AATC) were grown in DMEM-high glucose medium (HyClone) supplemented with $10 \%(786-0)$ or 15\% (Hep3B) FBS (Atlanta Biologicals), $20 \mathrm{mM}$ HEPES buffer $\mathrm{pH} 7.4,1 \mathrm{mM}$ sodium pyruvate, $100 \mathrm{U} / \mathrm{ml}$ penicillin and $100 \mu \mathrm{g} / \mathrm{ml}$ streptomycin (Invitrogen). Hypoxic experiments performed in a dedicated incubator (Coy Laboratory Products Inc) containing $1 \% \mathrm{O}_{2}, 5 \% \mathrm{CO}_{2}$ and balance $\mathrm{N}_{2}$. Compounds were added in DMSO ( $1 \%$ final).

RT-PCR. Cells were collected with Trizol (Invitrogen), and total RNA was extracted using the RNeasy Mini Kit (Qiagen). Following DNase treatment, cDNA was synthesized using SuperScript II Reverse Transcriptase (Invitrogen). Quantitative real time RT-PCR was performed in triplicate using iTaq SYBR Green Supermix with ROX (Bio-Rad) with the 7900HT detection system and software Prism (Applied Biosystems, Inc.). Data were analyzed using the comparative $\mathrm{C}_{\mathrm{T}}$ method ${ }^{51}$, and expression was normalized to cyclophilin B. The data for each gene are the mean of three values determined from three independently harvested sets. The following primer sets were used to amplify Cyclophilin B: (TGCCATCGCCAAGGAGTAG; TGCACAGACGGTCACTCAAA),HIF- $2 \alpha$ (GCGACAATGACAGCTGACAA; CAGCATCCCGGGACTTCT), EPO (GAGGCCGAGAATATCACGACGGG; 
TGCCCGACCTCCATCCTCTTCCAG), HIF- $1 \alpha$ (TGCCACATCATCACCA TATAGAGA; TCCTTTTCCTGCTCTGTTTGG), PGK1 (TTAAAGGGAAGC GGGTCGTTA; TCCATTGTCCAAGCAGAATTTGA) and VEGF1 (CTAC CTCCACCATGCCAAGTG; TGATTCTGCCCTCCTCCTTCT).

Coimmunoprecipitation. Nuclear protein extraction and co-IP experiments were performed as previously reported ${ }^{52}$. The following antibodies were used for immunoblot analysis: anti-HIF- $1 \alpha$ mouse monoclonal antibody (BD Biosciences; cat. no. 610959; 1:400 dilution); anti-EPAS/HIF-2 $\alpha$ mouse monoclonal antibody (Novus Biological; NB 100-132; 1:400 dilution) and anti-ARNT/HIF-1 $\beta$ mouse monoclonal antibody (Santa Cruz Biotechnology; sc-17811; $1: 800$ dilution).

Chromatin Immunoprecipitation (ChIP). Experiments were performed as described ${ }^{53}$ using the ChIP-IT Express Enzymatic Kit (Active Motif) according to the manufacturer's protocol. ChIP assays were carried out using normal mouse IgG (Santa Cruz Biotechnology; cat. no. \# 2027; 1-2 $\mu \mathrm{g} / \mathrm{ml}$ ), anti-HIF- $2 \alpha$ mouse monoclonal antibody (Novus Biologicals; NB 100-132; $2 \mathrm{mg} / \mathrm{ml}$ ) or anti-HIF- $1 \alpha$ mouse monoclonal antibody (BD Biosciences; cat. no. 610958; $2 \mathrm{mg} / \mathrm{ml}$ ). Genomic DNA, precipitated from a single $15-\mathrm{cm}$ plate for each treatment and maintained in parallel to those samples used in gene expression analyses, was analyzed by $\mathrm{qPCR}$ using the primers for a human EPO enhancer amplicon (ACTCCTGGCAGCAGTGCAGC; CCCTCTCCTTGATGACAATCTCAGC). The captured genomic DNA was normalized and compared between samples as previously reported ${ }^{26,53}$.

786-0 metabolic stability assays. 786-0 cells from ATCC (Manassas, VA) were plated at 5,000 cells per well in $50 \mu \mathrm{L}$ in 96-well plates and allowed to adhere overnight in standard RPMI growth medium containing 10\% FBS, $2 \mathrm{mM}$ glutamine, $1 \times$ penicillin-streptomycin, $10 \mathrm{mM}$ HEPES, $1 \mathrm{mM}$ sodium pyruvate and $1 \times$ nonessential amino acids (all purchased from Life Technologies, Grand Island, NY). Compound 2 was dissolved in DMSO at $2 \mathrm{mM}$, further diluted to $4 \mu \mathrm{M}$ in $\mathrm{HI}$ medium and added to the cells in $50 \mu \mathrm{L}$ so that the final compound concentration was $2 \mu \mathrm{M}$. Two additional wells containing compound and no cells were plated to serve as time $0\left(C_{0}\right)$ and endpoint solvent control $\left(C_{\mathrm{ep}}\right)$. The cells were then placed in a $37^{\circ} \mathrm{C}, 5 \% \mathrm{CO}_{2}$ incubator. At each time point, the medium was collected, the cells were washed once with PBS, and the wash was added to the media, and finally the cells were trypsinized, and the contents were added to the tube containing medium and PBS. An equal volume of methanol was added to lyse the cells and precipitate proteins. The samples were incubated for $10 \mathrm{~min}$ at RT and then spun at $15,000 \mathrm{~g}$ for $5 \mathrm{~min}$ in a microcentrifuge. The supernatant was analyzed by LC-MS/MS. Analytical methods were developed for 2 using an Applied Biosystems (Foster City, CA) 3200-QTrap, a combination triple quadrupole-ion trap instrument. The parent ion and the two most prominent daughter ions were followed to confirm compound identity, although only the most abundant daughter was used for quantification. An Agilent (Santa Clara, CA) ZORBAX XDB-C18 column, 5- $\mu$ m packing $4.6 \mathrm{~mm} \times 50 \mathrm{~mm}$ size, was used for chromatography.

A modified method ${ }^{54}$ was used for determination of metabolic stability half-life by substrate depletion. Briefly, a 'percentage recovered' number was calculated for the $C_{0}$ and $C_{\mathrm{ep}}$ samples plated in medium only to control for compound-related issues such as solubility and stability in the assay medium. This value was obtained by dividing the $C_{\text {ep }}$ LC-MS/MS peak area by the $C_{0}$ peak area and multiplying by 100 . Typically, acceptable values are between $70-140 \%{ }^{55}$, although half-lives are reported for compounds with lower percentage recovered values. A 'percentage remaining' value was used to assess metabolic stability of a compound over time. The LC-MS/MS peak area of the incubated sample at each time point was divided by the LC-MS/MS peak area of the time $0\left(T_{0}\right)$ sample and multiplied by 100 . The natural log (ln) of the percentage of compound remaining was then plotted versus time (in min), and a linear regression curve was plotted going through the $y$ intercept at $\ln (100)$. The metabolism of some compounds fail to show linear kinetics at a later time point, so those time points are excluded. The half-life $\left(t_{1 / 2}\right)$ was calculated as $t_{1 / 2}=0.693 /$ slope.

Additional chemical synthetic information and chemical characterization data are provided in the Supplementary Note.

43. Johnson, B.A. \& Blevins, R.A. NMRView: a computer program for the visualization and analysis of NMR data. J. Biomol. NMR 4, 603-614 (1994).

44. Otwinowski, Z. \& Minor, W. Processing of X-ray diffraction data collected in oscillation mode. Methods Enzymol. 276, 307-326 (1997).

45. Adams, P.D. et al. PHENIX: a comprehensive Python-based system for macromolecular structure solution. Acta Crystallogr. D Biol. Crystallogr. 66, 213-221 (2010).

46. Schüttelkopf, A.W. \& van Aalten, D.M. PRODRG: a tool for high-throughput crystallography of protein-ligand complexes. Acta Crystallogr. D Biol. Crystallogr. 60, 1355-1363 (2004).

47. Emsley, P. \& Cowtan, K. Coot: model-building tools for molecular graphics. Acta Crystallogr. D Biol. Crystallogr. 60, 2126-2132 (2004).

48. Chen, V.B. et al. MolProbity: all-atom structure validation for macromolecular crystallography. Acta Crystallogr. D Biol. Crystallogr. 66, 12-21 (2010).

49. Winn, M.D. et al. Overview of the CCP4 suite and current developments. Acta Crystallogr. D Biol. Crystallogr. 67, 235-242 (2011).

50. Martí-Renom, M.A. et al. Comparative protein structure modeling of genes and genomes. Annu. Rev. Biophys. Biomol. Struct. 29, 291-325 (2000).

51. Bookout, A.L. \& Mangelsdorf, D.J. Quantitative real-time PCR protocol for analysis of nuclear receptor signaling pathways. Nucl. Recept. Signal. 1, e012 (2003).

52. Scheuermann, T.H., Yang, J., Zhang, L., Gardner, K.H. \& Bruick, R.K. Hypoxia-inducible factors Per/ARNT/Sim domains: structure and function. Methods Enzymol. 435, 3-24 (2007).

53. Chen, R., Dioum, E.M., Hogg, R.T., Gerard, R.D. \& Garcia, J.A. Hypoxia increases sirtuin 1 expression in a hypoxia-inducible factor-dependent manner. J. Biol. Chem. 286, 13869-13878 (2011).

54. McNaney, C.A. et al. An automated liquid chromatography-mass spectrometry process to determine metabolic stability half-life and intrinsic clearance of drug candidates by substrate depletion. Assay Drug Dev. Technol. 6, 121-129 (2008).

55. Drexler, D.M. et al. An automated high throughput liquid chromatographymass spectrometry process to assess the metabolic stability of drug candidates. Assay Drug Dev. Technol. 5, 247-264 (2007). 